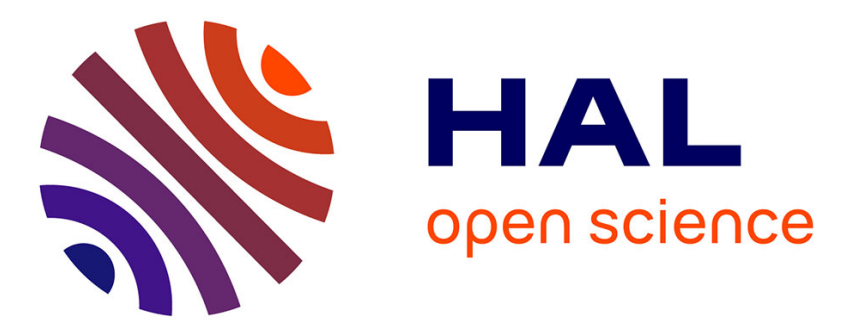

\title{
Submerged freeze gripper to manipulate Micro-objects.
}

Beatriz C. Lopez Walle, Michaël Gauthier, Nicolas Chaillet

\section{To cite this version:}

Beatriz C. Lopez Walle, Michaël Gauthier, Nicolas Chaillet. Submerged freeze gripper to manipulate Micro-objects.. IROS'06., Oct 2006, Beijing, China. pp.784-789, 10.1109/IROS.2006.282630 . hal00333570

\section{HAL Id: hal-00333570 \\ https://hal.science/hal-00333570}

Submitted on 23 Oct 2008

HAL is a multi-disciplinary open access archive for the deposit and dissemination of scientific research documents, whether they are published or not. The documents may come from teaching and research institutions in France or abroad, or from public or private research centers.
L'archive ouverte pluridisciplinaire HAL, est destinée au dépôt et à la diffusion de documents scientifiques de niveau recherche, publiés ou non, émanant des établissements d'enseignement et de recherche français ou étrangers, des laboratoires publics ou privés. 


\title{
Submerged Freeze Gripper to Manipulate Micro-objects
}

\author{
Beatriz López Walle, Michaël Gauthier and Nicolas Chaillet \\ Laboratoire d'Automatique de Besançon - UMR CNRS 6596 - ENSMM - UFC \\ 24, rue Alain Savary - 25000 Besançon, France \\ Email: \{blopez,gauthier,chaillet\}@ens2m.fr, Telephone: (33) (0) 381408801, Fax: (33) (0) 381402809
}

\begin{abstract}
Manipulating microscopic objects with the necessary dexterity still remains a very challenging task. In this paper, we propose a freeze gripper able to manipulate micro-objects in an innovative way, i.e. in submerged surroundings. We first review the influences of dry and liquid media on contact forces and non contact forces. This comparative analyse clearly shows the interest of the liquid medium. A survey of different microhandling systems based on the use of ice is also given. Then submerged microgripper with frozen water as an active end-effector is proposed as a promising new approach for manipulating low thermal conductive micro-objects. A prototype using the Peltier effect has been numerically modeled and developed. It is described in the paper. Experimental results validate the cooling and warming of the freeze gripper. A generic micromanipulation task is the purpose of further work.
\end{abstract}

\section{INTRODUCTION}

The demand for manipulating and assembling objects whose typical size is between one micrometer and one millimeter (usually corresponding to the so-called micromanipulation range) increases every day. The greatest difficulties in a micromanipulation task lie in its efficiency, reliability and precision of handling. All these criteria are strongly related to the interaction with the manipulated object [1]. At the present time, no repeatable and reliable micromanipulator exists for objects whose typical dimension is under 100 micrometers. At this scale, it is well known that the volumic forces are negligible compared to the surface forces, which affect the micromanipulation task and especially the release of the micro-object [2]. The development of novel methods to perform reliable micromanipulations under $100 \mu \mathrm{m}$ is thus particularly relevant.

In this paper we propose to manipulate and assembly artificial micro-objects at this scale being submerged in a liquid medium.

A comparative modeling of micromanipulation conditions in dry and liquid media of surface forces, contact forces (pull-off forces), and hydrodynamic forces shows that submerged micromanipulation has a lot of advantages under the limit of 100 micrometers [2], [3].

First, the van der Waals force, which is proportional to the Hamaker constant, is reduced in a liquid medium [4]. Moreover in such a media other typical forces appear: double layer force, solvation force, steric force. The sum of theses forces and the van der Waals force is exposed by the extended DVLO theory [5], [6]. In most cases these forces are repulsive and thus reduce the effect of the attractive van der Waals force.

Secondly, the electrostatic force is directly proportional to the charge density. This one depends on the triboelectrification and conductivity of the medium. The water, especially ionic water, has higher dielectric constant and better electric conductivity than the air. Consequently, electrostatic perturbations are greatly reduced in submerged conditions.

Third, the capillary force between an object and a substrate is induced by the surface between the liquid and the air near to the object. This surface disappears in immersion, so the capillary force is cancelled in a submerged medium [2], [3].

Fourth, the pull-off force represents the force necessary to break the contact surface between two objects. This force is proportional to the surface energy between two objects. This surface energy decreases when the objects are submerged [4]. Consequently, the pull-off force is reduced in liquid compared to air.

Finally, inertial effects are very small in the microworld, therefore the accelerations of the micro-objects are usually very high and then their velocity is able to increase in a very short time jumping very rapidly out of the work area and causing its loss. In most cases, velocity limitation in the submerged microworld depends on hydrodynamic physical limitation, but not on inertial physical limitation. Consequently, the hydrodynamic force drastically limits the maximal velocity of the micro-objects and then significantly reduces their loss rate. However, the movement of the microgripper tip can also lead to a significant hydrodynamic force on micro-objects. In this way, the hydrodynamic force induces a limitation of the maximum velocity of the microgripper end-effectors to avoid disturbances. Nevertheless, it was shown in a previous work that the maximum experimental effector velocity can still stay high even in water (e.g. $\left.1 \mathrm{~mm} \cdot \mathrm{s}^{-1}\right)$ compared to the typical size of the object manipulated $(50 \mu \mathrm{m})$.

This comparative analysis between air and liquid medium shows the interest of submerged micromanipulations. As previously mentioned, contact 
and non contact forces are reduced in liquid while hydrodynamic force significantly increases. Both phenomena are able to reduce respectively the electrostatic and adhesion perturbations, and the loss of micro-objects. First experimental comparative micromanipulation tests confirm the benefits of liquids. However, other experiments in course will allow validating entirely this concept and establish the accuracy expected for releasing inside liquid. In fact, the reliability of the release task remains a critical problem for so small objects.

In the present paper, a micromanipulation strategy adapted to the liquid medium is proposed. The liquid environment is exploited to generate an ice microsurface. The adhesive properties of the ice allow holding, manipulation and assembling of micro-objects. Moreover, as previously described we take advantages of the liquid medium to reduce critical adhesion: when the ice is thawed, it naturally disappears in the aqueous surroundings. Until now, our experiments have been performed in water. This principle has potentially many applications for the microassembly of miniaturized optical, mechanical and electrical components [1].

The section II presents a brief overview of several kinds of manipulation strategies which lead us to propose the use of an ice gripper principle in water. Then we describe the experimental device in section III and its modeling in section IV. At last, section V presents experimentations.

\section{Micromanipulation Strategies}

In this section, micromanipulation strategies are given, especially to study the release phase. It led us to propose the submerged freeze gripper as a promising approach.

\section{A. Gripping Strategies}

Numerous grip principles already exist for handling and assembling micro-objects. Above all, they must be able to catch, move, and release the manipulated object. Catching and moving micro-objects can be generally accomplished, but releasing them with a high reliability and a good accuracy still remains a critical problem [7]. We distinguish three strategies, presented in Fig. 1, to tackle adhesion forces during the release phase.

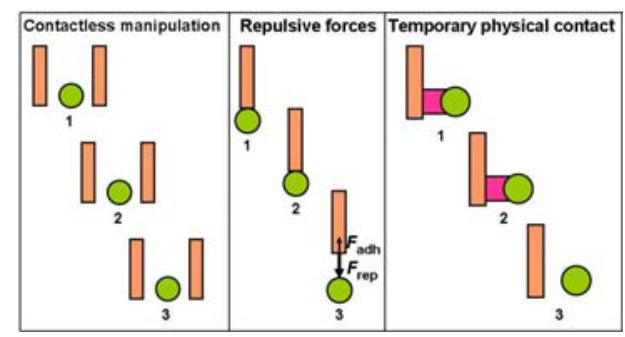

Fig. 1. Release strategies to overcome the adhesion forces.

The first one is the contactless manipulation. In this manipulation strategy, the gap between the object and the gripper always remains larger than the action range of adhesion forces. So, the handling task is not influenced by them. The common principle for contactless grippers is levitation that can be applied by different strategies such as the laser trapping [8] and the dielectrophoresis [9]. All of them need particular conditions for the objects (shape, size, material, etc.) and the surroundings (low temperature chambers, clean rooms, etc.).

In the second strategy, the manipulator system releases the micro-object generating repulsive forces $F_{\text {rep }}$. The electrostatic handling [10] and the release by acceleration principles [7] use repulsive forces bigger than adhesion forces $F_{a d h}$ to detach the object from the gripper. Unfortunately most often the final position of the object can not be totally controlled.

A temporary physical contact between the gripper and the object is used in the third strategy. The capillary, or surface tension, based gripper illustrates it [10]. In this manipulator system, a liquid droplet became a liquid bridge between the gripper and the object. The surface tension forces are used to handle the object. To release it the liquid bridge is evaporated, eliminating the physical contact. However, capillary forces generally do not disappear completely and other strategies must be combined, like the use of adhesive substrates [10], [11].

We choose the last principle (temporary physical medium between the object and the gripper) to develop the submerged freeze gripper. The handling procedure appears simple. First, the gripper approaches the object without touching it. Then, an ice microsurface is generated at the tip of the gripper freezing just a small part of the object or completely covering it. The object is handled. For the release phase, freezing is stopped, the ice microsurface thaws and the object is liberated. As the gripper and the object are submerged, the melted ice surface mixes with the medium, so capillary forces have no influence.

\section{B. Use of the Ice in Micromechatronics}

The ice in micromechatronics systems has two main applications: microfluidics and micromanipulation in air medium. In the first one, ice valves close or open a liquid flow in a microchannel [12], [13]. A thermoelectric device, such as the Peltier junction, is used to freeze the working fluid running inside the channel. Thereby the flow is blocked by the ice formation.

The adhesive properties of ice are also used for manipulating micro-objets in the air [1], [11], [14]. The cryogenic grippers based either on the Joule-Thompson effect [1], or on the Peltier effect [11], [14], freeze the water forming a thin ice film that connects the gripper and the object. The adhesive force generated by the ice allows us to pick up the object. The release of the object is generally done by stopping freezing or heating the gripper which thaws the ice. 
These cryogenic grippers look flexible and well-adapted to their applications. However, all of them work in air. To generate ice, the water is provided by a separated mechanism. In addition, particular conditions either environmental (low temperature and low humidity) or mechanical (release on adhesive surface) are needed. Finally, the size of the objects manipulated is bigger than 200 micrometers.

Despite the drawbacks cryogenic grippers are a very promising approach. They warrant a reliable grip with no surface damaging, provide high holding forces without introducing additional stresses in the object and the handling process can be designed as almost independent of shape and material properties.

In order to overcome the drawbacks we propose an innovative way which consists of the use of such a principle but totally submerged in a liquid medium.

\section{Experimental Device}

We proposed and developed a submerged freeze gripper based on Peltier effect. In this section, we describe the whole system principle. Then we present the physical characteristics of the prototype.

\section{A. Submerged Freeze Gripper Principle}

Cryogenic grippers presented above work in the air medium, so the water required to generate ice is supplied by an external device. The submerged freeze gripper utilizes the liquid environment to create an ice microsurface to catch objects with a low thermal conductivity and manipulate them. The cooling energy for freezing water is provided by the Peltier thermoelectric components.

The Peltier effect is a very well known principle for heating and cooling. Peltier elements consist of bismuth telluride $\mathrm{p}$ and $\mathrm{n}$-doped semiconductors connected electrically in series and thermally in parallel. As described by the Peltier effect, if the element is connected to direct current it generates a current-proportional heat flow that leads to a temperature difference between the two sides. If the electrical current is inverted, the direction of the heat generation and absorption inside the Peltier element is inversed too. So on, a Peltier module has two faces: a cold face and a hot face that needs a heat sink to extract the heat from the cold face.

The submerged freeze system, as shown in Fig. 2, consists on two Peltier module stages. The first one is a Peltier micromodule named MicroPelt $(\mu P)$. The gripper is directly attached to its cold side. So the MicroPelt can cool the gripper and consequently generate the ice microsurface on its acting part. The convection heat flow produced by the increasing of the temperature of the MicroPelt's hot face is so important than it could instead warm up the system (liquid, gripper and Peltier module) to cool it. We connected thus a second Peltier element to decrease the temperature at the hot face of the MicroPelt. The second Peltier module, that we called MiniPeltier $(m P)$, guarantees a constant temperature on the MicroPelt's heat sink. The MiniPeltier and its heat sink stay in air so that the heat is dissipated outside the water, in the air. The end-effector and the MicroPelt are completely submerged, so they require an electrical isolation.

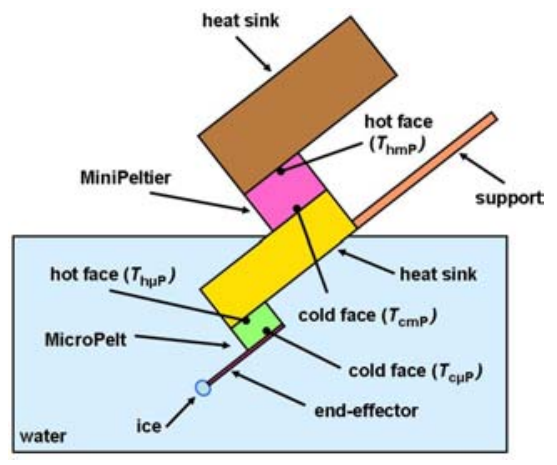

Fig. 2. Submerged freeze system principle.

\section{B. Physical and Technical Characteristics}

The experimental device shown in Fig. 3, consists of two Peltier modules with their respective heat sinks, a support for the MicroPelt's electrical connection and a Nickel tip.

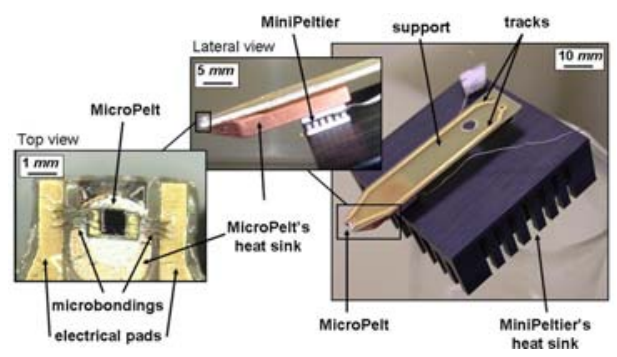

Fig. 3. Experimental freeze system.

The MicroPelt (Infineon Technologies AG), whose dimensions are $720 \times 720 \times 428 \mu^{3}$, is fastened to a copper heat sink with silver glue that provides excellent thermal conductivity. The MiniPeltier's (Melcor FC0.6-18$05)$ dimensions are $6.2 \times 6.2 \times 2.4 \mathrm{~mm}^{3}$. Its cold face is fixed to the MicroPelts' heat sink while its hot face is fixed to an Aluminium heat sink.

The micro-end-effector is built in a $1500 \times 3500 \times 20$ $\mu \mathrm{m}^{3}$ Nickel sheet. The shape is designed to take advantage of the whole cooling power of the MicroPelt. Its body covers thus all the surface of the cold face of the MicroPelt, as well as the tip that corresponds to the size of the objects that will be gripped.

Because of the dimensions of the MicroPelt, the electrical connections between the MicroPelt's electrical contacts and a support specially designed for this purpose, have been established using microbonding technology. 


\section{Microgripper Modeling}

The Peltier modules are controlled by electrical current. The thickness of the ice surface on the end-effector tip, Thick $_{i c e}$, depends thus on both the electrical current of the MicroPelt, $i_{\mu P}$, and the electrical current of the MiniPeltier, $i_{m P}$ (Fig. 4). The objective of this section is to determine the optimal values of these electrical currents.

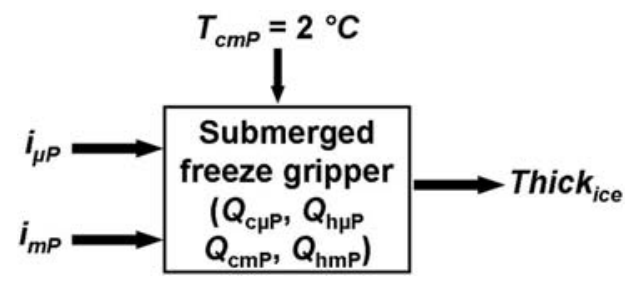

Fig. 4. Acting parameters in microgripper modeling.

The Peltier modules represent the active elements of the submerged freeze gripper. Every Peltier module provides a cooling capacity $\left(Q_{c}\right)$, which depends on its geometric and physical characteristics. The cooling capacity expression includes the Peltier effect $\left(-\alpha T_{c} i\right)$, the Joule effect $\left(R i^{2} / 2\right)$ and the heat flow through heat conductivity $(k \Delta T)$, which can be written as:

$$
Q_{c}=-\alpha T_{c} i+\frac{R i^{2}}{2}+k \Delta T
$$

where $\alpha$ is the Peltier coefficient, $R$ is the electrical resistance, $k$ is the thermal conductivity coefficient of the module, $i$ is the electrical current supplied, $T_{c}$ is the temperature at the cold face, and $\Delta T$ is the difference of temperature between the two faces.

The heating capacity $Q_{h}$ is:

$$
Q_{h}=\alpha T_{h} i+\frac{R i^{2}}{2}-k \Delta T
$$

where $T_{h}$ is the temperature at the hot face.

Table I gives the physical parameters for the MicroPelt and the MiniPeltier.

TABLE I

Physical parameters for the Peltier elements

\begin{tabular}{|l|c|c|}
\hline Coefficient & $\begin{array}{c}\text { MicroPelt } \\
(\mu P)\end{array}$ & $\begin{array}{c}\text { MiniPetier } \\
(m P)\end{array}$ \\
\hline Peltier coefficient $\alpha(\mathrm{V} / \mathrm{K})$ & $1.0 \cdot 10^{-3}$ & $7.0 \cdot 10^{-3}$ \\
Electrical resisitance $R(\Omega)$ & 0.2 & 1.158 \\
Heat conductivity $k(W / m K)$ & 0.0082 & 0.017 \\
\hline
\end{tabular}

For the thermal study as well as for the examination of the working principles in order to optimize the design of the gripper, the whole submerged ice gripper system has been modeled using the finite elements software (FEM) COMSOL Multiphysics 3.2.
The FEM calculation was divided in two parts: in the first one, we calculated the optimal electrical current for every Peltier module using static modeling; in the second one, we examined the dynamical behaviour of the system.

\section{A. Static Modeling}

The cooling capacity $Q_{c}$ of the Peltier elements is a parabolic function of the current $i$ according to (1).

In the case of the MicroPelt, an optimal current $i_{\mu P}^{*}$ exists when this cooling capacity $Q_{c \mu P}$ is maximal and when the temperature on its cold side $T_{c \mu P}$ is minimal. So, for $i_{\mu P}=i_{\mu P}^{*}$ the temperature on the tip surface is also minimal.

The MicroPelt injects the cooling capacity to the gripper in order to create the ice surface as we explained below. To warrant the maximal cooling of the MicroPelt, the temperature of its hot face must be slightly above $0^{\circ} \mathrm{C}$. Because of the latter, the temperature of the MiniPeltier's cold side $T_{c m P}$ keeps at $2^{\circ} \mathrm{C}$. This temperature is sufficiently close to $0^{\circ} \mathrm{C}$ but it prevents it from freezing the heat sink of the MicroPelt.

To determine the optimal current of the MicroPelt, the temperature of the cold side of the MiniPeltier $T_{c m P}$ is fixed at $2^{\circ} C$, the behaviour of the surroundings medium is simulated by the FEM calculation and we range $i_{\mu P}$ to obtain the minimal temperature $T_{c \mu P}$. Fig. 5 shows that this optimal current $i_{\mu P}^{*}$ is $1.1 \mathrm{~A}$.

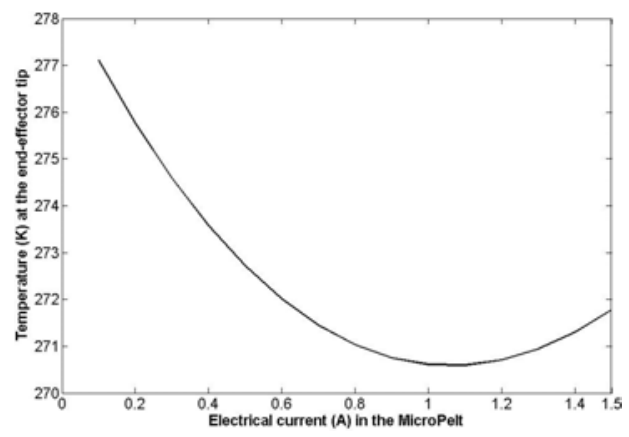

Fig. 5. Temperature at the active surface of the gripper as a function of MicroPelt's electrical current.

Knowing the optimal current of the MicroPelt, we can establish the optimal current in the MiniPeltier $i_{m P}^{*}$ that must warranties the temperature of its cold side $T_{c m P}$ at $2^{\circ} C$ as we referred below. In Fig. 6 we note that this temperature is reached at $i_{m P}^{*}=0.8 \mathrm{~A}$.

\section{B. Dynamical Modeling}

Dynamical performances of the system can be modeled using both optimal electrical currents $i_{\mu P}^{*}$ and $i_{m P}^{*}$, and (1) and (2) for the two thermoelectric elements.

The dynamical modeling of the submerged freeze gripper allows us first to design the geometry of the Nickel end-effector to obtain the best cooling flow, secondly to observe ice surface generation and its forms on the active 


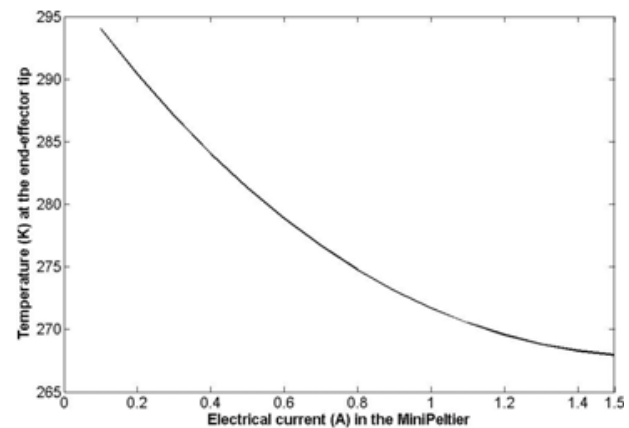

Fig. 6. Temperature at cold surface of the MiniPeltier as a function of MiniPeltier's electrical current.

surface and, finally to obtain the cycle times of the system.

So first, as the thermal performance of the gripper was a critical parameter, the Nickel end-effector has been designed using FEM with the aim of optimizing heat distribution while keeping a reduced size of the tip. Fig. 7 presents the design of the end-effector used in the experiments.

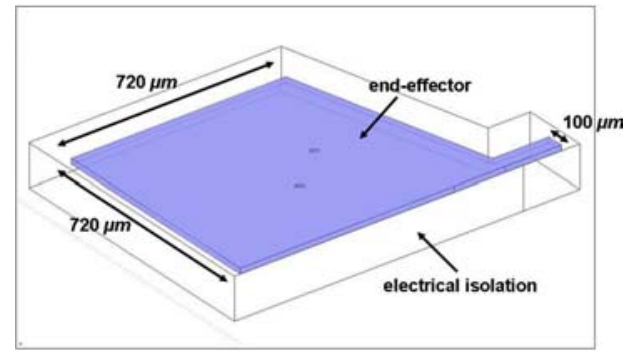

Fig. 7. Nickel microtip design.

Secondly, in order to study the working of the gripper, the ice generation on the active surface of the gripper was modeled at different times applying the optimal electrical currents. We observed that the ice was ellipsoidal in shape and depends on cooling time. In Fig. 8 we see the ice shape at the tip after 1 second when the temperature of the water surrounding was $2^{\circ} \mathrm{C}$.

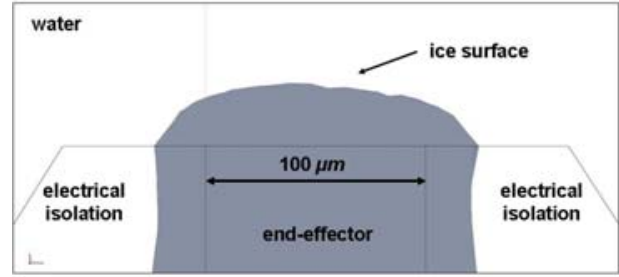

Fig. 8. Ice surface at the tip after 1 second of cooling.

The release of the object is done either reversing the electrical current of the Peltier module or simply cancelling it. Nevertheless, reversing the direction of the electrical current produces the inversion of the heat flow inside the
Peltier module. We cancel thus the current of MicroPelt in order to avoid the overheating of its cold face.

Finally, the dynamical modeling allowed us to obtain both maximal cycle time and stable working cycle of the gripper. The electrical current of the MiniPeltier stays constant at its optimal value $i_{m P}^{*}$. The current through the MicroPelt $i_{\mu P}$ ranges between zero and its optimal value $i_{\mu P}^{*}=1.1 \mathrm{~A}$. The maximal cycle time for a freezing-thawing cycle was 0.2 seconds (Fig. 9) and the stable working cycle is found at 2 seconds (Fig. 10).

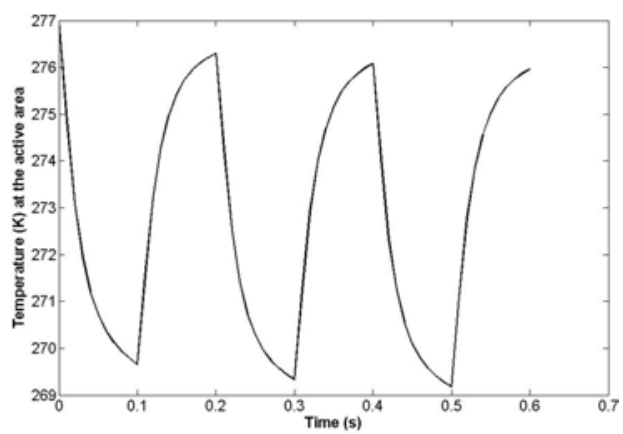

Fig. 9. Temperature at the active area of the microgripper as a function of time. Maximal cycle time of the gripper.

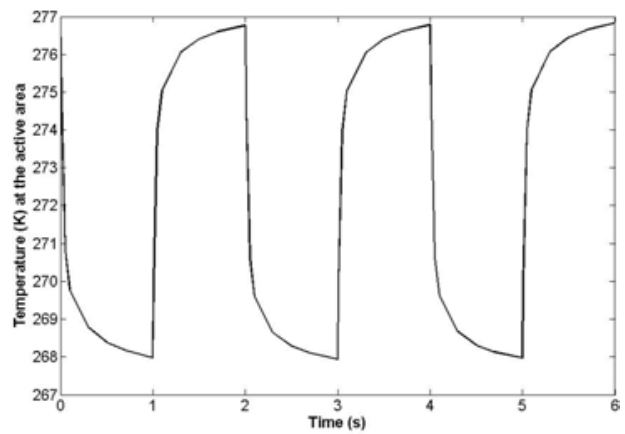

Fig. 10. Temperature at the active area of the microgripper as a function of time. Stable cycle time of the gripper.

Numerical modeling was used to study and validate our submerged freeze gripper. Modeling interactivity between the end-effector and different material objects confirms that manipulation is better for objects with a low thermal conductivity. Moreover, optimal parameters for the experimental micromanipulations were obtained. In addition, the working cycle found $(0.3 \mathrm{~s})$ is very short and can be compared with the cycle times of mechanical tweezers and vacuum grippers [11].

\section{ExPERIMENTAL VALIDATION}

The process steps and the cycle time were observed thanks to the experimentations.

Fig. 11 shows the three phases of the process: (a) precooling, (b) cooling under $0^{\circ} \mathrm{C}$ and (c) heating over $0^{\circ} \mathrm{C}$. 
In fact, the MiniPeltier is activated at $t=0 \mathrm{~s}$ to decrease the temperature of the MicroPelt's heat sink during the precooling phase (a). The MicroPelt is then activated and the temperature on the end-effector tip decreases under $0^{\circ} \mathrm{C}$ as shown in phase (b). Finally, if the electrical current of the MicroPelt is cancelled, as observed in phase (c), the temperature on the end-effector tip increases over $0^{\circ} C$.

In this experimentation, the current $i_{m P}$ is set constant at $i_{m P}^{*}=0.8 A$. To test the validity of the microgripper, the current $i_{\mu P}$ is first limited to $0.2 A$. The temperature is measured with a microthermocouple. Its effects as a source of thermal conduction are not considered.

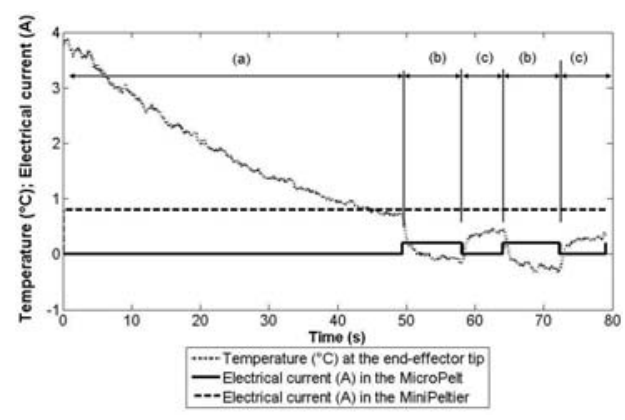

Fig. 11. Temperature at the active area of the microgripper, electrical current in the MicroPelt, and electrical current in the MiniPeltier as a function of time.

The working cycle of the MicroPelt is presented in Fig. 12. We appreciate that the temperature at the active area of the microgripper decreases under $0^{\circ} \mathrm{C}$ in $0.5 \mathrm{~s}$ after the activation of the MicroPelt. However, after cancelling MicroPelt's electrical current, the end-effector takes more than $0.6 s$ to pass over $0^{\circ} \mathrm{C}$.

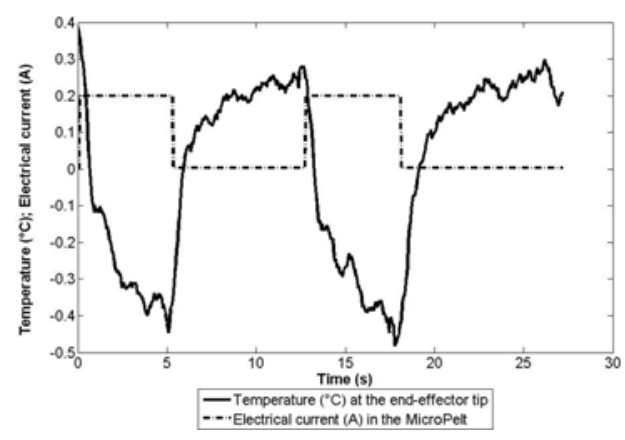

Fig. 12. Temperature at the active area of the microgripper and electrical current in the MicroPelt as a function of time.

Experimentations allow thus validating the thermal principle of the submerged freeze gripper. Controlling both Peltier modules temperature through their electrical currents and micro-object manipulations will be the objective of further work.

\section{Conclusion}

Reliable manipulations for micro-objects sized under the $100 \mu \mathrm{m}$ require novel gripping strategies. The analysis of contact forces, distance forces and hydrodynamic forces shows that performing micromanipulations in a liquid medium is more interesting than dry micromanipulations. We develop an innovative micromanipulation strategy adapted to the aquatic medium. Effectively, the submerged freeze gripper presented in this paper, exploits the liquid medium to generate an ice microsurface to manipulate micro-objects. Furthermore, it takes advantages of the environment mixing the micro-ice formation in this aquatic medium when it thaws to release the object, overcoming thus the capillary force. Modeling the acting principle based on Peltier effect brings out process parameters like optimal electrical currents and cycle times. The principle has been also validated by experimentations. Further works will focus on manipulating micro-objects, improving the control of the temperature and thickness of the ice microsurface, and the miniaturization of the system to manipulate micro-objects sized under the $50 \mu \mathrm{m}$.

\section{REFERENCES}

[1] J. Liu, Y.-X. Zhou, and T.-H. Yu, "Freeze tweezer to manipulate mini/micro objects," Journal of Micromechanics and Microengineering, vol. 14, no. 2, pp. 269-276, February 2004.

[2] M. Gauthier, B. López-Walle, and C. Clévy, "Comparison between micro-objects manipulations in dry and liquid mediums," in In Proc. of the 6th CIRA, Espoo, Finland, June 2005.

[3] M. Gauthier et al., "Forces analyses for micromanipulations in dry and liquid media," Journal of Micromechatronics, accepted November 2005.

[4] J. Israelachvili, Intermolecular and surfaces forces, A. Press, Ed., 1991.

[5] Z. Xu and R. H. Yoon, "The role of hydrophobic interactions in coagulation," Journal of Colloid Interface Science, vol. 44, no. 132, pp. 532-541, 1989.

[6] , "A study of hydrophobic coagulation," Journal of Colloid Interface Science, vol. 45, no. 134, pp. 427-434, 1990.

[7] D. S. Haliyo and S. Régnier, "Advanced applications using micromad, the adhesion based dynamic micro-manipulator," in In Proc. of AIM, Port Island, Japan, July 2003, pp. 880-885.

[8] F. Arai et al., "Synchronized laser micromanipulation of microtools for assembly of microbeads and indirect manipulation of microbe," in In Proc. of IROS, Las Vegas, USA, October 2003, pp. 2121-2126.

[9] A. Rosenthal and J. Voldman, "Dielectrophoretic traps for single-particle patterning," Biophysical Journal, vol. 88, March 2005.

[10] P. Lambert, "A contribution to microassembly: a study of capillary forces as a gripping principle," Ph.D. dissertation, Université Libre de Bruxelles, 2004.

[11] S. Droz et al., "New generation of grippers for the manipulation of miniaturized components," in Proc. of Mechatronics, Besançon, France, October 2001, pp. 572-575.

[12] L. Gui and J. Liu, "Ice valve for a mini/micro flow channel," Journal of Micromechanics and Microengineering, vol. 14, pp. 242-246, November 2004.

[13] R. P. Welle and B. S. Hardy, "Fabrication and charaterization of fast-acting peltier-actuated microvalves," in In Proc. of the 9th MicroTAS, Boston, USA, October 2005, pp. 1078-1080.

[14] G. Seliger, J. Stephan, and S. Lange, "Hydroadhesive gripping by using peltier effect," in In Proc. of IMECE, Florida, USA, November 2000 , pp. 3-8. 